\title{
Evaluation of Estimators for Hybrid GNSS-Terrestrial Localization in Collaborative Networks
}

\author{
Daniel Medina $^{1}$, Lars Grundhöfer ${ }^{2}$ and Niklas Hehenkamp ${ }^{2}$
}

\begin{abstract}
Global Navigation Satellite Systems (GNSS) constitute the cornerstone for outdoor positioning, which is essential information for prospective automated vehicles. The combination of GNSS with terrestrial ranging, for instance in the form of 5G or UWB, will make accurate positioning a reality even in urban canyon scenarios where GNSS is likely to fail. Thus, hybrid GNSS-terrestrial localization in collaborative networks has become a hotspot for the research community. This paper discusses the Cramér-Rao Bound (CRB) as lower bound for location estimates and evaluates two snapshot estimators, one deterministic and the other Bayesian, for distributed and centralized localization in cooperative networks. The performance of the estimators is evaluated with respect to the presented CRB in a simulated network of mobile and anchor agents, and the role played by agent-to-agent and agentto-anchor ranging is discussed.
\end{abstract}

\section{INTRODUCTION}

In challenging urban environments, obstacles can obstruct the visibility of Global Navigation Satellite Systems (GNSS) satellites. If GNSS satellites are not in line of sight, GNSSbased services may lose reliability or even fail completely. A higher elevation mask might diminishes reflection and multipath effects, at the cost of a reduced satellite geometry [1], [2]. Recent approaches on collaborative hybrid localization (GNSS and another ranging technology) have been presented, showcasing promising results on reliable positioning in obstructive scenarios [3]-[8]. While Inertial Measurement Units, optical sensors and other sensors can support positioning in these cases [9]-[13], this work focuses on signals of opportunity that enable the obtainment of pseudo-ranges between devices.

The current development and research efforts in 5G cellular networks suggest that future cellular user equipment will feature device-to-device ranging capabilities on top an improved device-to-infrastructure ranging features [14][18]. The combined use of device-to-device and deviceto-infrastructure ranging can provide a positioning solution whose accuracy and reliability depends on the density of devices and infrastructure elements. Moreover, future millimeter-wave systems will implement angle of departure (AoD) and angle of arrival (AoA) estimation through beamforming with the help of highly directional, steerable and compact antenna arrays [19]. The demand and importance

\footnotetext{
${ }^{1}$ Daniel Medina is with the Institute of Communications and Navigation, German Aerospace Center (DLR), Germany and with Computer Science Engineering Department, Universidad Carlos III de Madrid, Spain.

${ }^{2}$ Lars Grundhöfer and Niklas Hehenkamp are with the Institute of Communications and Navigation, German Aerospace Center (DLR), Germany. emails: \{daniel.ariasmedina, lars.grundhoefer, niklas.hehenkamp\}@dlr.de
}

for location awareness in diverse applications increases, especially in wireless networks. Localization methods developed within the wireless networking domain can benefit GNSS based positioning to increase its accuracy. Likewise GNSS can support localization within wireless networks if the involved devices feature GNSS positioning. A collaborative approach can benefit the overall localization problem.

We distinguish between two types of nodes. Nodes whose states are unknown are referred to as agents. Nodes which have known states at all times are referred to as anchors. Both agents and anchors may be mobile. [6] demonstrate that GNSS positioning can be improved by providing accurate range estimates between a GNSS equipped agent and an anchor at a well known position. The scenario covers localization techniques provided in wireless networks considering solely agent-to-anchor ranging into account. However, the collaboration of agents within a network provides the possibility of agent to agent ranging.

This paper presents the signal model for localization estimation in hybrid GNSS-terrestrial collaborative networks and the associated estimation bound is discussed. Lower estimation bounds constitute a fundamental tool to assess the minimal performance, in terms for instance of mean squared error (MSE), an estimator can achieve. Bounds can be categorized as deterministic or Bayesian [20]. This work considers the first, for which the unknown parameters are considered deterministic and evaluate the locally best estimator performance [21]. In particular, we re-visit the Cramér-Rao Lower Bound (CLRB) derived for hybrid collaborative localization [3], and the importance of the different ranging modalities among the agents is discussed. Besides, this work examines the families of localization algorithms and evaluates the performance for snapshot estimation for deterministic and probabilistic methods, for positioning in collaborative networks. The experimentation is based on a synthetic collaborative network for which four agents tracking GNSS satellites exchange ranging information with each other, as well as with other two anchor nodes. The CRBs are derived for each of these agents to showcase the gain from each of the ranging modes, as well as to address the performance of the proposed positioning methods.

The rest of the paper is organized as follows. In Section 2 , the signal model for the hybrid localization in cooperative networks is introduced. Section 3 presents the CRB for the localization problem. Section 4 discusses the families of positioning algorithms. Section 5 presents the experimentation and discussion. Finally, outlook and future work are presented in Section 6. 


\section{Signal Model}

Let us consider a general cooperative network consisting of $A$ anchor nodes, whose positions $\mathbf{p}_{a}$ and clock biases are known, and $M$ agent nodes whose positions $\mathbf{p}_{m}$ and receiver clock biases $b_{m}$ are unknown. Besides, $S$ satellites can be tracked by the participating agents. Each agent $m$ can be subscribed to a particular number of anchors, communicate with certain other agents, and track a set of satellites. Thus, we denote with $\mathcal{A}_{m}, \mathcal{M}_{m}, \mathcal{S}_{m}$ the subset of anchors, agents and satellites, respectively, that an agent $m$ receives ranging from. The number of elements composing the aforementioned subsets is accordingly $A_{m}, M_{m}$ and $S_{m}$.

We can distinguish three kind of measurements, based on the range observed from agent $m$ to satellites, anchors or other agents:

$$
\begin{aligned}
\rho_{s \rightarrow m} & =\left\|\mathbf{p}_{s}-\mathbf{p}_{m}\right\|+b_{m}+\varepsilon_{s \rightarrow m}, \\
r_{a \rightarrow m} & =\left\|\mathbf{p}_{a}-\mathbf{p}_{m}\right\|+\varepsilon_{a \rightarrow m}, \\
r_{n \rightarrow m} & =\left\|\mathbf{p}_{n}-\mathbf{p}_{m}\right\|+\varepsilon_{n \rightarrow m},
\end{aligned}
$$

where $\mathbf{p}_{(\cdot)}, \varepsilon_{(\cdot) \rightarrow m}$ are respectively the position and observation noise for $(\cdot)=\left\{s \in \mathcal{S}_{m}, a \in \mathcal{A}_{m}, n \in \mathcal{M}_{m}\right\}$. The observation noises are assumed to be independent and to follow a zero-mean normal distribution

$$
\varepsilon_{(\cdot) \rightarrow m} \sim \mathcal{N}\left(0, \sigma_{(\cdot) \rightarrow m}^{2}\right) .
$$

For agent $m$, the vectors of unknowns $\mathbf{x}_{m}$ and observations $\mathbf{y}_{m}$ are expressed as follows

$$
\begin{aligned}
& \mathbf{x}_{m}=\left[\mathbf{p}_{m}^{\top}, b_{m}\right]^{\top}, \mathbf{x}_{m} \in \mathbb{R}^{D+1}, \\
& \mathbf{y}_{m}=\left[\boldsymbol{\rho}_{\mathcal{S}_{m}}^{\top}, \mathbf{r}_{\mathcal{A}_{m}}^{\top}, \mathbf{r}_{\mathcal{M}_{m}}^{\top}\right]^{\top}, \mathbf{y}_{m} \in \mathbb{R}^{S_{m}+A_{m}+M_{m}},
\end{aligned}
$$

where $D$ indicates the dimension of the position vector, depending on whether two- or three-dimensional localization is considered.

By stacking the vector of unknowns for each participating agent:

$$
\mathbf{X}=\left[\mathbf{x}_{1}, \ldots, \mathbf{x}_{M}\right], \mathbf{X} \in \mathbb{R}^{(D+1) M} .
$$

Similarly, the complete set of observations is formulated as

$$
\mathbf{Y}=\left[\boldsymbol{\rho}_{\mathcal{S}_{1}}^{\top}, \ldots, \rho_{\mathcal{S}_{M}}^{\top}, \mathbf{r}_{\mathcal{A}_{1}}^{\top}, \ldots, \mathbf{r}_{\mathcal{A}_{M}}^{\top}, \mathbf{r}_{\mathcal{M}_{1}}^{\top}, \ldots, \mathbf{r}_{\mathcal{M}_{M}}^{\top}\right]^{\top},
$$

with $\mathbf{Y} \in \mathbb{R}^{\sum_{i}\left(S_{i}+A_{i}+M_{i}\right)}$. The covariance matrix of the observations $\mathbf{R}$ is described as dispersion over such set of observations

$$
\mathbf{R} \triangleq \mathbb{D}(\mathbf{Y})=\left[\begin{array}{ll}
\mathbf{R}_{\rho} & \\
& \mathbf{R}_{r}
\end{array}\right],
$$

where $\mathbf{R}_{\rho}$ and $\mathbf{R}_{r}$ are the covariance matrices for the GNSS and terrestrial observations respectively, whose diagonal values agree with Eq. (4). In general, cross-correlation among observations could exist, especially for the GNSS case when nearby agents are tracking the same satellite. Also, NLOS and multipath effects at a certain location are suffered in a similar way accross the agents. However, these correlations are out of the scope of this work and therefore disregarded.

\section{CRB FOR COLLABORATIVE LOCALIZATION}

The CRB describes the lowest bound on the mean squared error (MSE) of any unbiased estimator. The bound can be described by the diagonal elements of the inverse of the Fisher Information Matrix (FIM) [20], [22]. Provided the FIM for the vector of unknown parameters $\mathbf{x}$, denoted as $\mathbf{F}(\mathbf{x})$, the associated $\mathrm{CRB}$ is

$$
C R B_{\mathbf{x}} \triangleq \mathbf{F}(\mathbf{x})^{-1} \preceq \mathbb{E}\left\{(\mathbf{x}-\hat{\mathbf{x}})(\mathbf{x}-\hat{\mathbf{x}})^{\top}\right\},
$$

where $\hat{\mathbf{x}}$ is an unbiased estimate and $\mathbb{E}$ is the expectation operator. For agent $m$, its associated FIM can be obtained as

$$
\mathbf{F}_{m}=-\mathbb{E}\left\{H_{m}\left(\Lambda_{m}\left(\mathbf{x}_{m}\right)\right)\right\},
$$

with $H_{m}$ the Hessian operator for the vector $\mathbf{x}_{m}$ and $\Lambda_{m}$ the sum of the log-likelihood function for observations. Considering first the non-cooperative (GNSS-only) case, we define

$$
\Lambda_{s \rightarrow m}\left(\mathbf{x}_{m}\right)=\sum_{s \in \mathcal{S}_{m}} \mathcal{L}\left(\rho_{m \rightarrow s} \mid \mathbf{x}_{m}\right) .
$$

Taking into account all $M$ agents, the FIM for GNSS is

$$
\mathbf{F}_{\text {gnss }}=\left[\begin{array}{ccc}
\mathbf{F}_{1} & & \\
& \ddots & \\
& & \mathbf{F}_{M}
\end{array}\right] .
$$

For the hybrid GNSS-terrestrial cooperative localization, we extend the log-likelihood function to

$$
\begin{aligned}
\Lambda_{m}\left(\mathbf{x}_{m}\right) & =\sum_{s \in \mathcal{S}_{m}} \mathcal{L}\left(\rho_{s \rightarrow m} \mid \mathbf{x}_{m}\right) \\
& +\sum_{a \in \mathcal{A}_{m}} \mathcal{L}\left(r_{a \rightarrow m} \mid \mathbf{x}_{m}\right) \\
& +\sum_{m \in M} \sum_{n \in \mathcal{M}_{m}} \mathcal{L}\left(r_{n \rightarrow m} \mid \mathbf{x}_{m}, \mathbf{x}_{n}\right)
\end{aligned}
$$

Using (11), we can split up the calculation for each agent as

$$
\mathbf{F}_{m}=\mathbf{F}_{s \rightarrow m}+\mathbf{F}_{a \rightarrow m}+\mathbf{F}_{n \rightarrow m},
$$

while for the multi-agent case, the FIM is

$$
\mathbf{F}_{M}=\mathbf{F}_{\text {gnss }}+\mathbf{F}_{\text {coop }}
$$

where

$$
\mathbf{F}_{\text {coop }}=\left[\begin{array}{lll}
\mathbf{F}_{a \rightarrow 1} & & \\
& \ddots & \\
& & \mathbf{F}_{a \rightarrow M}
\end{array}\right]+\mathbf{F}_{\text {coop }, n m} .
$$

The different matrices are defined as

$$
\begin{aligned}
\mathbf{F}_{s \rightarrow m} & =-\mathbb{E}\left\{\mathbf{H}_{m}\left(\Lambda_{s \rightarrow m}\left(\mathbf{x}_{m}\right)\right)\right\}, \\
\mathbf{F}_{a \rightarrow m} & =-\mathbb{E}\left\{\mathbf{H}_{m}\left(\Lambda_{a \rightarrow m}\left(\mathbf{x}_{m}\right)\right)\right\}, \\
\mathbf{F}_{\text {coop }, n m} & =-\mathbb{E}\left\{\mathbf{H}_{m n}\left(\Lambda_{n \rightarrow m}\left(\mathbf{x}_{m}, \mathbf{x}_{n}\right)\right)\right\} .
\end{aligned}
$$

with

$$
\begin{aligned}
\Lambda_{a \rightarrow m}\left(\mathbf{x}_{m}\right) & =\sum_{a \in \mathcal{A}_{m}} \mathcal{L}\left(r_{a \rightarrow m} \mid \mathbf{x}_{m}\right) \\
\Lambda_{n \rightarrow m}\left(\mathbf{x}_{m}, \mathbf{x}_{n}\right) & =\sum_{m \in M} \sum_{n \in \mathcal{M}_{m}} \mathcal{L}\left(r_{n \rightarrow m} \mid \mathbf{x}_{m}, \mathbf{x}_{n}\right)
\end{aligned}
$$


where $\mathbf{H}_{m n}$ is the cross Hessian matrix with dimension $\mathbf{H}_{m n} \in \mathbb{R}^{(D+1) M,(D+1) M}$.

Based on the split up, we derive the FIMs described in Eq. (15). For the non-cooperative case, the associated FIM is

$$
\mathbf{F}_{s \rightarrow m}=\left[\begin{array}{cc}
\mathbf{F}_{\mathbf{p}_{m}} & \mathbf{F}_{\mathbf{p}_{m}, b_{m}} \\
\mathbf{F}_{\mathbf{p}_{m}, b_{m}}^{\top} & F_{b_{m}}
\end{array}\right] \succeq 0
$$

where

$$
\begin{aligned}
\mathbf{F}_{\mathbf{p}_{m}} & =\sum_{s \in \mathcal{S}_{m}} \frac{1}{\sigma_{s \rightarrow m}^{2}} \mathbf{q}_{s m} \mathbf{q}_{s m}^{\top}, \\
\mathbf{F}_{\mathbf{p}_{m}, b_{m}} & =\sum_{s \in \mathcal{S}_{m}} \frac{1}{\sigma_{s \rightarrow m}^{2}} \mathbf{q}_{s m}, \\
F_{b_{m}} & =\sum_{s \in \mathcal{S}_{m}} \frac{1}{\sigma_{s \rightarrow m}^{2}}
\end{aligned}
$$

where $\mathbf{q}_{i m}$ defines the unit-length steering vector between $\mathbf{p}_{i}$ and $\mathbf{p}_{m}$ as $\mathbf{q}_{i m}=\frac{\mathbf{p}_{i}-\mathbf{p}_{m}}{\left\|\mathbf{p}_{i}-\mathbf{p}_{m}\right\|}$.

Since there are no cross correlations for the measurement of the anchor points, $\mathbf{F}_{a \rightarrow m}$ can be described as

$$
\mathbf{F}_{a \rightarrow m}=\left[\begin{array}{cc}
\mathbf{F}_{\mathbf{p}_{m}} & 0 \\
0 & 0
\end{array}\right]
$$

with

$$
\mathbf{F}_{\mathbf{p}_{m}}=\sum_{a \in \mathcal{A}_{m}} \frac{1}{\sigma_{a \rightarrow m}^{2}} \mathbf{q}_{a m} \mathbf{q}_{a m}^{T} .
$$

In order to derive $\mathbf{F}_{\text {coop }, n m}$, we need to utilize the cross Hessian matrix

$$
\mathbf{H}_{m n}=\left[\begin{array}{ccc}
\frac{\partial^{2}}{\partial \mathbf{x}_{1} \partial \mathbf{x}_{1}} & \cdots & \frac{\partial^{2}}{\partial \mathbf{x}_{1} \partial \mathbf{x}_{M}} \\
\vdots & \ddots & \vdots \\
\frac{\partial^{2}}{\partial \mathbf{x}_{M} \partial \mathbf{x}_{1}} & \cdots & \frac{\partial^{2}}{\partial \mathbf{x}_{M} \partial \mathbf{x}_{M}}
\end{array}\right]
$$

Applying (19) we get the block matrix in the form

$$
\mathbf{F}_{\mathrm{coop}, n m}=\left[\begin{array}{ccccccc}
\mathbf{F}^{\prime}{ }_{1} & \mathbf{0} & \mathbf{K}_{12} & \mathbf{0} & \cdots & \mathbf{K}_{1 M} & \mathbf{0} \\
\mathbf{0}^{\top} & 0 & \mathbf{0}^{\top} & 0 & \cdots & \mathbf{0}^{T} & 0 \\
\vdots & & & \ddots & & \vdots & \\
\mathbf{K}_{M 1} & \mathbf{0} & & \cdots & & \mathbf{F}^{\prime}{ }_{M} & \mathbf{0} \\
\mathbf{0}^{\top} & 0 & & \cdots & & \mathbf{0}^{\top} & 0
\end{array}\right]
$$

where $\mathbf{0}$ is the $D$-size zero vector, and

$$
\begin{gathered}
\mathbf{F}_{m}^{\prime}=\sum_{n \in \mathcal{M}_{m}} \frac{1}{\sigma_{n \rightarrow m}^{2}} \mathbf{q}_{n m} \mathbf{q}_{n m}^{\top}, \\
\mathbf{K}_{m n}= \begin{cases}-\frac{1}{\sigma_{n \rightarrow m}^{2}} \mathbf{q}_{n m} \mathbf{q}_{n m}^{\top}, & \text { if } n \in \mathcal{M}_{m} \\
0 & \text { otherwise. }\end{cases}
\end{gathered}
$$

The generic expressions described above can be applied to a particular network configuration for the estimate of the associated FIM. The inversion of Eq. (16) expresses the CRLB for the estimate of $\mathbf{X}$.

\section{LOCALIZATION ALGORITHMS}

The problem of localization for collaborative networks can be formulated from different perspectives and, similarly, algorithms for its resolution can be distinguished in various categories. This work discusses two classifications: centralized vs. decentralized estimation and deterministic vs. Bayesian approaches.

\section{A. Centralized vs. Distributed}

Centralized estimation of the collaborative localization problem requires the participating agents sending their sensed observations from satellites and other agents to a central node in charge of computing the complete set of unknowns $\mathbf{X}$ simultaneously. Thus, the minimization problem can be expressed as next

$$
\begin{aligned}
\hat{\mathbf{X}}=\arg \min _{\mathbf{X}} & \sum_{m=1}^{M}\left\{\sum_{a \in \mathcal{A}_{m}} \frac{1}{\sigma_{a \rightarrow m}^{2}}\left(r_{a \rightarrow m}-\left\|\mathbf{p}_{a}-\mathbf{p}_{m}\right\|^{2}\right)\right. \\
& +\sum_{s \in \mathcal{S}_{m}} \frac{1}{\sigma_{s \rightarrow m}^{2}}\left(\rho_{s \rightarrow m}-\left\|\mathbf{p}_{s}-\mathbf{p}_{m}\right\|^{2}-b_{m}\right) \\
& \left.+\sum_{n \in \mathcal{M}_{m}} \frac{1}{\sigma_{n \rightarrow m}^{2}}\left(r_{n \rightarrow m}-\left\|\mathbf{p}_{n}-\mathbf{p}_{m}\right\|^{2}\right)\right\} .
\end{aligned}
$$

In this case, the technical challenge lies on the capabilities of the involved agents to forward their respective data to the central node and the posterior dispatching of the obtained localization solution to the pertinent agent. Regarding mathematical complexity, notice that Eq. (26) comprises a nonlinear, non-convex optimization problem for which a closedform solution is not known. Centralized estimation brings clear assets: first, the privacy between users is respected, since they would not exchange information with each other; second, centralized methods exploit information from the entire network and are supposed to yield more accurate location estimation [8].

Distributed or decentralized localization approaches imply that each agent estimates its own location. Thus, the associated optimization problem is as follows

$$
\begin{aligned}
\hat{\mathbf{x}}_{m}=\arg \min _{\mathbf{x}_{m}} & \left\{\sum_{s \in \mathcal{S}_{m}} \frac{1}{\sigma_{s \rightarrow m}^{2}}\left(\rho_{s \rightarrow m}-\left\|\mathbf{p}_{s}-\mathbf{p}_{m}\right\|^{2}-b_{m}\right)\right. \\
& +\sum_{a \in \mathcal{A}_{m}} \frac{1}{\sigma_{a \rightarrow m}^{2}}\left(r_{a \rightarrow m}-\left\|\mathbf{p}_{a}-\mathbf{p}_{m}\right\|^{2}\right) \\
& \left.+\sum_{n \in \mathcal{M}_{m}} \frac{1}{\sigma_{n \rightarrow m}^{2}}\left(r_{n \rightarrow m}-\left\|\mathbf{p}_{n}-\mathbf{p}_{m}\right\|^{2}\right)\right\} .
\end{aligned}
$$

Distributed estimation has the advantage of being more robust to failures, since the computation is spread over the network. However, distributed approaches requires agents to interchange location information, which might compromise the individual privacy. Moreover, the exchanged position between mobile agents might be incorrect, leading to further estimation errors. Fig. 1 provides a pictorial example of the aforementioned issues for the distributed localization of agent 4, based on the depicted network. For simplicity, 

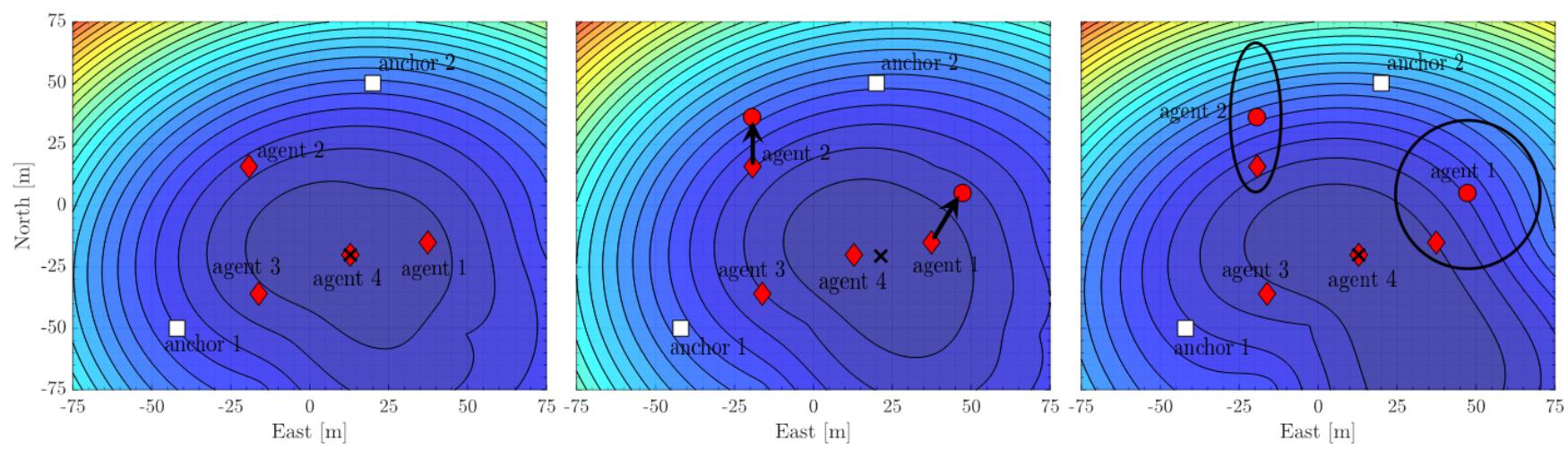

Fig. 1. Example of distributed collaborative localization for agent 4. The contour plots depicts the least square (LS) cost function for whose the position minimizing the optimization is indicated with a cross. On the left, it is depicted the ideal case, where the location of the other agents is known. On the center, agents 1 and 2 communicate that their respective positions wrongly correspond to the red circles. On the right, the position of agents 1 and 2 is wrong, but an estimate of the variance of their position is known.

satellite information is not considered in this example and the ranging observations are free from noise. On Fig. 1 (left), the positioning information provided from agents 1,2 and 3 is completely accurate. Thus, the position minimizing the least square (LS) adjustment coincides with the reality. Fig. 1 (center) depicts the situation for which the positions of agents 1 and 2 are wrong, but information on their location bias is not included in the estimation. Therefore, the LS minimization for the position of agent 4 is wrongly estimated. Finally, Fig. 1 (right) shows a similar situation, where the locations of agent 3 and 4 is wrongly estimated, but information of the covariance of their respective position solutions in incorporated into the estimation. Therefore, the observations of these two "malicious" agents are de-weighted with respect to the range measurements to agent 2 and the two anchor nodes and the final localization of agent 4 is correct.

\section{B. Deterministic vs. Bayesian}

Deterministic approaches consider the state estimation to be a deterministic but unknown vector. Generally, these approaches compute a solution in a snapshot manner, i.e. no prior knowledge on the unknown parameters is incorporated and solely observations received at a particular time are considered. An extensive number of deterministic approaches have been proposed for localization in collaborative networks, including simulated annealing [23], [24], semidefinite programming [25]-[28] or parallel projection methods [29], [30]. Nevertheless, classical Maximum Likelihood (ML) estimation remains one of the preferred alternatives for deterministic localization, given its asymptotic optimality and mathematical simplicity [31], [32]. ML aims at finding the estimate that minimizes Eq. (26) or (27), for centralized or distributed problems respectively. Due to the non-linear non-convex nature of the problem at hand, the optimality (and even convergence) of ML estimates is subject to the choice of the initial point. Steepest descent, shift-cutting and Gauss-Newton (GN) are often the preferred algorithms applied to drive the ML estimate [33], [34]. Since GNSS- based positioning is generally accurate enough to serve as starting search point, localization in hybrid GNSS-terrestrial networks shall not be especially sensitive to convergence issues with gradient-directed searches in ML.

In probabilistic localization approaches, the state estimates are considered random variables whose probability density function is to be estimated. Generally, the unknowns are considered to follow a normal distribution whose parameters -mean and variance- are to be inferred [35]-[37]. While snapshot estimation is generally solved with one of the deterministic methods aforementioned, Bayesian approaches constitute the base for recursive estimation, which allows incorporating the motion model for the tracked agents and integrating series of measurements over time [38]-[41].

\section{Proposed Bayesian Localization}

This work proposes an iterative Bayesian estimator, inspired by the works on Iterated Extended Kalman Filter [42], [43]. Its application to centralized and distributed localization in cooperative networks is discussed. Particularly for de-centralized estimation, some form of message passing between agents is required, where each agent provides information on their position $\hat{\mathbf{p}}_{n}$ and associated covariance matrix $\hat{\mathbf{P}}_{n}$ for the position estimate. Considering the agent-to-agent ranging observation in Eq. 3, the noise term needs to account for the uncertainty on the position estimate of agent $n$ as:

$$
\tilde{\sigma}_{n \rightarrow m}=\sigma_{n \rightarrow m}+\sqrt{\operatorname{tr}\left(\hat{\mathbf{P}}_{n}\right)}
$$

where $\operatorname{tr}(\cdot)$ is the matrix trace operator. The described noise model for agent-to-agent measurements was also illustrated in Fig. 1 (right), where the uncertainty for the positions of agents 1 and 2 was accommodated into the estimation. In general, GNSS positioning can serve as initialization point for estimating both centralized or distributed approaches. GNSS positioning is generally realized by applying leastsquares adjustments [44], [45]. An overview of the algorithm is provided in Alg. 1. 

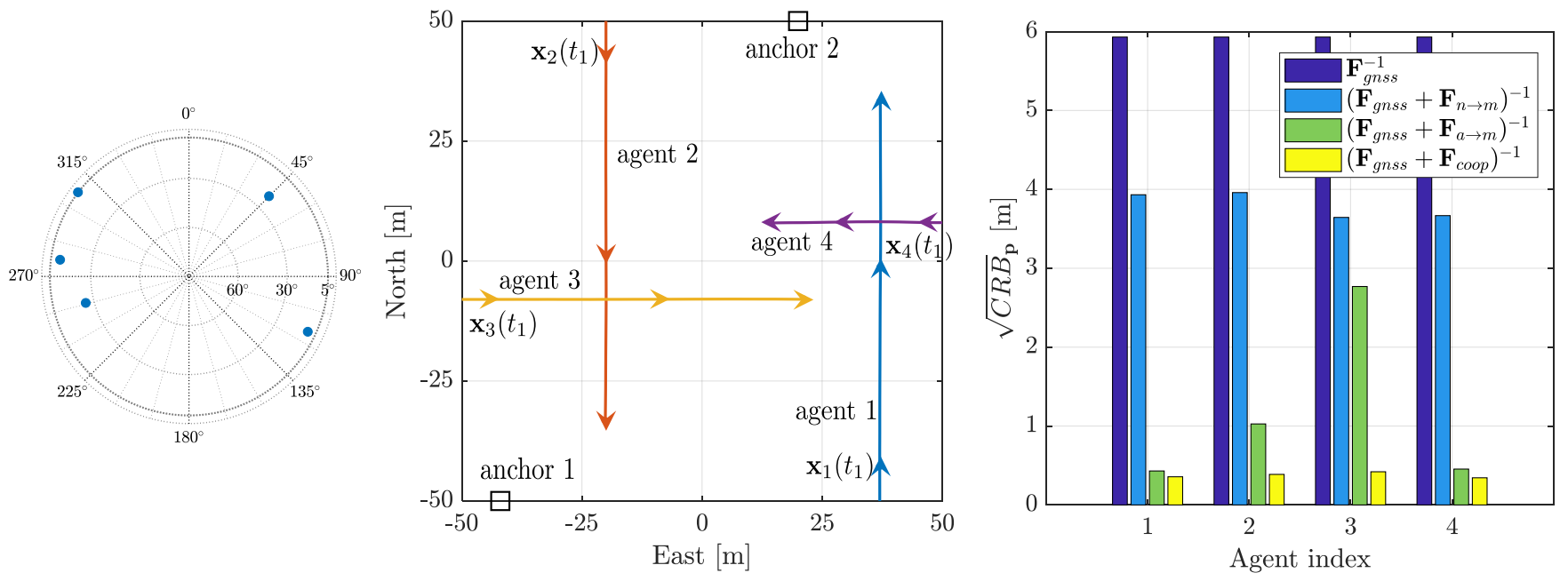

Fig. 2. On the left, Sky plot for the GNSS satellites tracked by the mobile agents proposed in the exemplary network. On the center, network for collaborative positioning based on hybrid GNSS and terrestrial range measurements. On the left, CRB for position estimates for each of the agents, based on the observation type used: GNSS-only (dark blue), GNSS+ranging to other agents (light blue), GNSS+ranging to anchor (green), GNSS+ranging to anchors and agents (yellow).

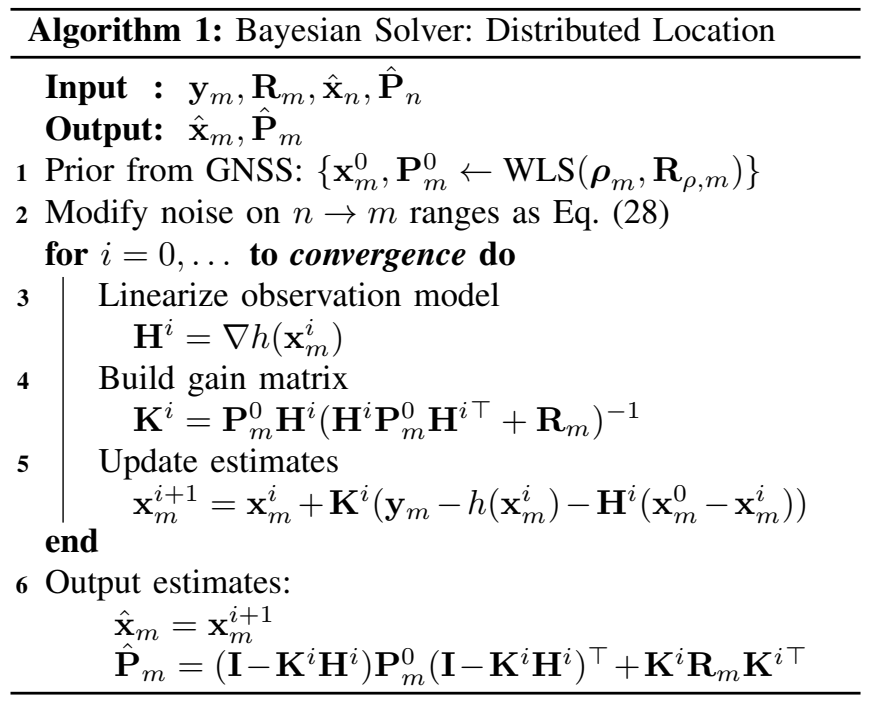

\section{EXPERIMENTATION}

To provide insight into the hybrid GNSS-terrestrial collaborative localization problem, the network depicted in Fig. 2 (center) serves as example. Within an area of 50 by 50 meters, the network consists of four mobile agents, two anchor nodes and the five satellites shown in the sky plot of Fig. 2 (left). For the sake of simplicity, the network is assumed to be fully connected -i.e., all agents measure ranges to the two anchor nodes, to the other agents and track the same number of GNSS satellites. Despite the agents being mobile, note that the estimation is realized in any case in a snapshot manner, so that the resulting estimates of the compared algorithm methods can be compared to the deterministic bounds described in Sect. III. The noise on GNSS range observations are considered independent and identically distributed (iid), according to a zero-mean normal distribution with a deviation $\sigma_{\rho}=5$ meters. Noise on terres- trial range measurements also obeys the iid condition and is withdrawn from a normal distribution with $\sigma_{r}=0.30 \mathrm{~m}$. For the characterization of the proposed positioning estimators, the results shown next are obtained after averaging over 1000 Monte Carlo experiments.

First, the estimation bounds for the different ranging modalities is discussed. Fig. 2 (right) depicts the square root of the trace for the CRB -taking solely the diagonal values related to the position estimate or, in other words, disregarding the clock bias. Fig. 2 (right) shows the CRB for localization based for the four mobile agents, corresponding to the time indicated with the (second) arrow on Fig. 2 (center). The ranging modes are indicated as follows: dark blue for GNSS-only, light blue for GNSS and agent-to-agent ranging, green for GNSS and agent-to-anchor and yellow for combining all ranging approaches. Since all agents track the five available satellites and the agent-to-satellite line-of-sight vector barely changes among agents, the GNSS-based CRB remains equal for all the agents. Also, as the network is fully connected, the localization gain by adding inter-agent ranging to satellite-based positioning barely changes across the agents. Due to the position of the agents being unknown, the advantage of agent-to-agent ranging might seem minimal compared to agent-to-anchor ranging. When compared to GNSS, the main positioning gain is related to anchor-tonode ranging, with the CRB being mostly dominated by the low-noise of the terrestrial ranging. However, on real-life situations and vehicular applications, an agent might not be able to range to multiple anchors and the relative geometry is then of great importance. An example of the role played by the agent-to-anchor geometry is illustrated for the agent 3 in Fig. 2 (center and right). At the studied time, agent 3 is almost in line with the two anchor nodes, having therefore a very poor geometry. This is reflected on its CRB for GNSS + anchor ranging, which is considerably higher than for the remaining agents. The benefits of GNSS + agent-to-all 
terrestrial ranging is depicted, being the positioning CRB significantly lower than GNSS-only. Notice again, that the communication to the anchors is the driver component.

Next, the positioning performance of the proposed estimators is addressed. To support the discussion of the importance of the different ranging approaches, Fig. 3 depicts the information ellipses at $90 \%$ confidence interval for the horizontal positioning errors for a distributed ML estimator using, respectively, GNSS (dark blue), GNSS and agent-toagent (light blue), GNSS and agent-to-anchor (green) and GNSS and terrestrial (yellow) ranging observations. The results resemble what one might expect from the estimated CRB above, although the integration of the anchor ranging does not reach the lower bound from the CRB, making manifest the non-optimality of the estimates.

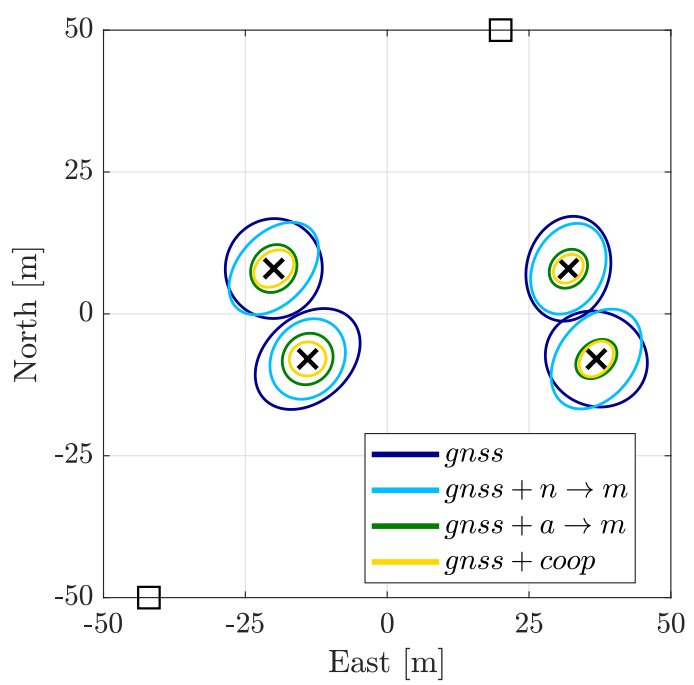

Fig. 3. Information ellipses for the horizontal positioning errors at $90 \%$ confidence interval.

Fig. 4 depicts the CRB for the horizontal positioning problem based on GNSS observations (solid blue) and for hybrid GNSS terrestrial ranges (solid orange). The root mean squared errors (RMSE) of the derived ML and Bayesian estimators are shown as well. For GNSS-only positioning, the ML is known to be an optimal estimator, since the nonlinearity and simplicity of the problem is reduced. This is demonstrated empirically on our Monte Carlo experiment, since the RMSE of the solver lies directly on the associated CRB. On the other hand, the proposed estimators are not optimal, with their performance being a meter worse than the theoretical lower bound. This is most likely due to the nonlinearity and non-convexity of the problem, for which the estimator is subject to lie on a local minimum instead of the global one. Nonetheless, the Bayesian approach appears quite promising, since it retains a performance similar to the ML one, while still being compatible with tracking approaches. When incorporating information of the dynamical model of an agent and recursively filtering their noisy observations, Bayesian methods have a promising future for localization in collaborative networks.

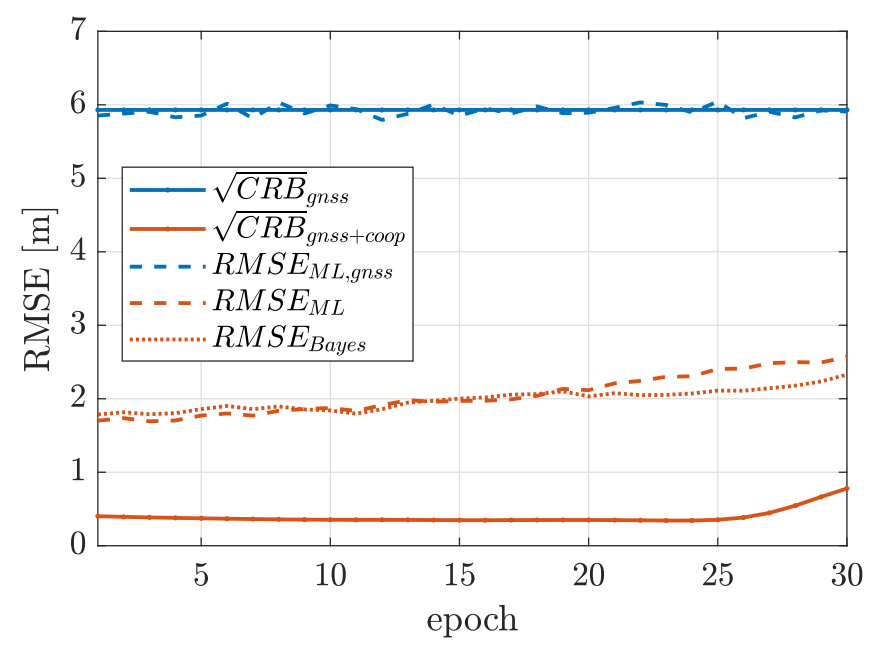

Fig. 4. CRB for the positioning of agent 1 over time, as well as the RMSE for the proposed ML and Bayesian methods. For reference, the CRB for GNSS-only and the associated RMSE for a ML estimator is shown in blue.

\section{OUTLOOK}

This paper presents the need for algorithms for localization estimation in hybrid GNSS-terrestrial collaborative networks, especially with the prospective application of $5 \mathrm{G}$ device-todevice communication. The localization problem is formally studied, from the point of view of the information theory, by presenting the Cramér-Rao bounds of the estimated. Besides, it is discussed the families of positioning algorithms, based on whether they are centralized or distributed, as well as deterministic or probabilistic. A simulated network serves as experimentation, allowing us to discuss the importance of agent-to-anchor compared to agent-to-agent ranging observations. Deterministic ML and Bayesian positioning methods are explored and evaluated against the corresponding CRB for horizontal positioning. Unfortunately, current solvers cannot reach the CRB lower bound and therefore, they are not optimal. Bayesian methods might be especially interesting for future works, since its application to recursive estimation is immediate and the incorporation of the dynamical model of the agents is a fundamental tool for precise and robust localization in cooperative networks.

\section{REFERENCES}

[1] P. J. G. Teunissen, R. Odolinski, and D. Odijk, "Instantaneous BeiDouGPS RTK positioning with high cut-off elevation angles," Journal of Geodesy, vol. 88, no. 4, pp. 335-350, dec 2013. [Online]. Available: https://doi.org/10.1007\%2Fs00190-013-0686-4

[2] J. M. Castro-Arvizu, D. Medina, and R. Ziebold, "Impact of Satellite Elevation Mask in GPS+Galileo RTK Positioning," in Proceedings of the 2020 International Technical Meeting of The Institute of Navigation. Institute of Navigation, feb 2020. [Online]. Available: https://doi.org/10.33012\%2F2020.17157

[3] F. Penna, M. A. Caceres, and H. Wymeersch, "Cramér-rao bound for hybrid GNSS-terrestrial cooperative positioning," IEEE Communications Letters, vol. 14, no. 11, pp. 1005-1007, nov 2010. [Online]. Available: https://doi.org/10.1109\%2Flcomm.2010.091310.101060

[4] G. Cueto-Felgueroso, F. Grec, J. del Peral-Rosado, G. SecoGranados, C. Gentner, E. Staudinger, D. Bartlett, E. Serna, M. Azaola, E. Domínguez, A. Fernández, F. Blázquez, F. Mata, R. Prieto-Cerdeira, and L. Ries, "Hybridizing GNSS with sensors and terrestrial technologies for positioning in 5g," in Proceedings of the 
32nd International Technical Meeting of the Satellite Division of The Institute of Navigation (ION GNSS 2019). Institute of Navigation, oct 2019. [Online]. Available: https://doi.org/10.33012\%2F2019.17131

[5] A. Minetto and F. Dovis, "A theoretical framework for collaborative estimation of distances among gnss users," in 2018 IEEE/ION Position, Location and Navigation Symposium (PLANS). IEEE, 2018, pp. $1492-1501$.

[6] _ _ "On the information carried by correlated collaborative ranging measurements for hybrid positioning," IEEE Transactions on Vehicular Technology, vol. 69, no. 2, pp. 1419-1427, 2019.

[7] C. Gioia and D. Borio, "GNSS-based Cooperative Positioning: Approaches and Demonstration," in Proceedings of the 32nd International Technical Meeting of the Satellite Division of The Institute of Navigation (ION GNSS+ 2019). ION, 2019, pp. 3832-3846.

[8] R. M. Buehrer, H. Wymeersch, and R. M. Vaghefi, "Collaborative sensor network localization: Algorithms and practical issues," Proceedings of the IEEE, vol. 106, no. 6, pp. 1089-1114, jun 2018 [Online]. Available: https://doi.org/10.1109\%2Fjproc.2018.2829439

[9] C. Fernández-Prades, P. Closas, and J. Vila-Valls, "Nonlinear filtering for ultra-tight gnss/ins integration," in 2010 IEEE International Conference on Communications. IEEE, 2010, pp. 1-5.

[10] D. A. Medina, M. Romanovas, I. Herrera-Pinzón, and R. Ziebold, "Robust position and velocity estimation methods in integrated navigation systems for inland water applications," in 2016 IEEE/ION Position, Location and Navigation Symposium (PLANS). IEEE, 2016, pp. 491501.

[11] L. Grundhöfer and S. Gewies, "R-mode receiver development for medium frequency signals," Scientific Journals of the Maritime University of Szczecin, 2018. [Online]. Available: https://doi.org/10.1007\%2Fs00190-013-0686-4

[12] R. Ziebold, D. Medina, M. Romanovas, C. Lass, and S. Gewies, "Performance characterization of GNSS/IMU/DVL integration under real maritime jamming conditions," Sensors, vol. 18, no. 9, p. 2954 2018.

[13] G. A. Pagès and J. Vilà-Valls, "Robust TOA-Based Navigation under Measurement Model Mismatch in Harsh Propagation Environments,' in 2020 23rd International Conference on Intelligent Transportation Systems (ITSC). IEEE, 2020.

[14] L. Militano, G. Araniti, M. Condoluci, I. Farris, and A. Iera, "Deviceto-device communications for 5g internet of things," EAI Endorsed Transactions on Internet of Things, vol. 15, no. 1, pp. 1-15, 2015.

[15] R. M. Buehrer, H. Wymeersch, and R. M. Vaghefi, "Collaborative sensor network localization: Algorithms and practical issues," Proceedings of the IEEE, vol. 106, no. 6, pp. 1089-1114, 2018.

[16] H. Wymeersch, J. Lien, and M. Z. Win, "Cooperative localization in wireless networks," Proceedings of the IEEE, vol. 97, no. 2, pp. $427-$ 450, 2009.

[17] A. Dammann, R. Raulefs, and S. Zhang, "On prospects of positioning in 5g," in 2015 IEEE International Conference on Communication Workshop (ICCW). IEEE, 2015, pp. 1207-1213.

[18] M. Castillo-Lopez, P. Ludivig, S. A. Sajadi-Alamdari, J. L. SanchezLopez, M. A. Olivares-Mendez, and H. Voos, "A Real-Time Approach for Chance-Constrained Motion Planning With Dynamic Obstacles,' IEEE Robotics and Automation Letters, vol. 5, no. 2, pp. 3620-3625, 2020.

[19] R. Koirala, B. Denis, B. Uguen, D. Dardari, and H. Wymeersch, "Localization and throughput trade-off in a multi-user multi-carrier mm-wave system," IEEE Access, vol. 7, pp. 167 099-167 112, 2019.

[20] H. L. Van Trees and K. L. Bell, "Bayesian bounds for parameter estimation and nonlinear filtering/tracking," $A M C$, vol. 10, p. 12, 2007.

[21] D. Medina, J. Vilà-Valls, E. Chaumette, F. Vincent, and P. Closas, "Cramér-Rao bound for a mixture of real- and integer-valued parameter vectors and its application to the linear regression model," Submitted to Signal Processing, 2020.

[22] N. Kbayer, J. Galy, E. Chaumette, F. Vincent, A. Renaux, and P. Larzabal, "On lower bounds for nonstandard deterministic estimation," IEEE Transactions on Signal Processing, vol. 65, no. 6, pp. 1538-1553, 2016.

[23] A. A. Kannan, G. Mao, and B. Vucetic, "Simulated annealing based wireless sensor network localization." JCP, vol. 1, no. 2, pp. 15-22, 2006.

[24] _ "Simulated annealing based wireless sensor network localization with flip ambiguity mitigation," in 2006 IEEE 63rd Vehicular Technology Conference, vol. 2. IEEE, 2006, pp. 1022-1026.
[25] L. Doherty, L. El Ghaoui et al., "Convex position estimation in wireless sensor networks," in Proceedings IEEE INFOCOM 2001. Conference on Computer Communications. Twentieth Annual Joint Conference of the IEEE Computer and Communications Society (Cat. No. 01CH37213), vol. 3. IEEE, 2001, pp. 1655-1663.

[26] P. Biswas and Y. Ye, "Semidefinite programming for ad hoc wireless sensor network localization," in Proceedings of the 3rd international symposium on Information processing in sensor networks, 2004, pp. 46-54.

[27] P. Tseng, "Second-order cone programming relaxation of sensor network localization," SIAM Journal on Optimization, vol. 18, no. 1, pp. 156-185, 2007.

[28] R. M. Vaghefi and R. M. Buehrer, "Cooperative localization in nlos environments using semidefinite programming," IEEE Communications Letters, vol. 19, no. 8, pp. 1382-1385, 2015.

[29] T. Jia and R. M. Buehrer, "Collaborative position location for wireless networks using iterative parallel projection method," in 2010 IEEE Global Telecommunications Conference GLOBECOM 2010. IEEE, 2010, pp. 1-6.

[30] _ - "A set-theoretic approach to collaborative position location for wireless networks," IEEE Transactions on Mobile Computing, vol. 10, no. 9, pp. 1264-1275, 2010.

[31] J. J. Caffery, "A new approach to the geometry of toa location," in Vehicular Technology Conference Fall 2000. IEEE VTS Fall VTC2000. 52nd Vehicular Technology Conference (Cat. No. OOCH37152), vol. 4. IEEE, 2000, pp. 1943-1949.

[32] N. Patwari, A. O. Hero, M. Perkins, N. S. Correal, and R. J. O'dea, "Relative location estimation in wireless sensor networks," IEEE Transactions on signal processing, vol. 51, no. 8, pp. 2137-2148, 2003.

[33] C. T. Kelley, Iterative methods for optimization. SIAM, 1999.

[34] R. Zekavat and R. M. Buehrer, Handbook of position location: Theory, practice and advances. John Wiley \& Sons, 2011, vol. 27.

[35] D. Barber, Bayesian reasoning and machine learning. Cambridge University Press, 2012.

[36] P. Closas, C. Fernandez-Prades, and J. A. Fernandez-Rubio, "A bayesian approach to multipath mitigation in gnss receivers," IEEE Journal of Selected Topics in Signal Processing, vol. 3, no. 4, pp. 695-706, Aug 2009.

[37] D. Medina, K. Gibson, R. Ziebold, and P. Closas, "Determination of pseudorange error models and multipath characterization under signaldegraded scenarios," in Proceedings of the 31st International Technical Meeting of the Satellite Division of The Institute of Navigation (ION GNSS+ 2018), Miami, FL, USA, 2018, pp. 24-28.

[38] T. Lv, H. Gao, X. Li, S. Yang, and L. Hanzo, "Space-time hierarchicalgraph based cooperative localization in wireless sensor networks," IEEE Transactions on Signal Processing, vol. 64, no. 2, pp. 322-334, 2015.

[39] F. Yin, C. Fritsche, D. Jin, F. Gustafsson, and A. M. Zoubir, "Cooperative localization in wsns using gaussian mixture modeling: Distributed ecm algorithms," IEEE Transactions on Signal Processing, vol. 63, no. 6, pp. 1448-1463, 2015.

[40] S. Li, M. Hedley, and I. B. Collings, "New efficient indoor cooperative localization algorithm with empirical ranging error model," IEEE Journal on Selected Areas in Communications, vol. 33, no. 7, pp. 1407-1417, 2015

[41] F. Scheidt, D. Jin, M. Muma, and A. M. Zoubir, "Fast and accurate cooperative localization in wireless sensor networks," in 201624 th European Signal Processing Conference (EUSIPCO). IEEE, 2016, pp. 190-194.

[42] M. A. Skoglund, G. Hendeby, and D. Axehill, "Extended kalman filter modifications based on an optimization view point," in 2015 18th International Conference on Information Fusion (Fusion). IEEE, 2015, pp. 1856-1861

[43] M. A. Skoglund, F. Gustafsson, and G. Hendeby, "On iterative unscented kalman filter using optimization," in 2019 22th International Conference on Information Fusion (FUSION). IEEE, 2019, pp. 1-8.

[44] P. Teunissen and O. Montenbruck, Springer handbook of global navigation satellite systems. Springer, 2017.

[45] D. Medina, L. Ortega, J. Vilà-Valls, P. Closas, F. Vincent, and E. Chaumette, "A New Compact CRB for Delay, Doppler and Phase Estimation-Application to GNSS SPP \& RTK Performance Characterization," IET Radar Sonar \& Navigation, 2020. 